ARTICLE

\title{
Direct imaging and electronic structure modulation of moire superlattices at the 2D/3D interface
}

\author{
Kate Reidy (1) 1, Georgios Varnavides (1) 1,2,3,4,7, Joachim Dahl Thomsen ${ }^{1}$, Abinash Kumar', Thang Pham¹, \\ Arthur M. Blackburn (10 ${ }^{5}$, Polina Anikeeva (iD) ${ }^{1,2,6}$, Prineha Narang (1) ${ }^{3}$, James M. LeBeau (i) ${ }^{1}$ \& \\ Frances M. Ross (iD ${ }^{1 \times}$
}

The atomic structure at the interface between two-dimensional (2D) and three-dimensional (3D) materials influences properties such as contact resistance, photo-response, and highfrequency electrical performance. Moiré engineering is yet to be utilized for tailoring this 2D/ $3 D$ interface, despite its success in enabling correlated physics at 2D/2D interfaces. Using epitaxially aligned $\mathrm{MoS}_{2} / \mathrm{Au}\{111\}$ as a model system, we demonstrate the use of advanced scanning transmission electron microscopy (STEM) combined with a geometric convolution technique in imaging the crystallographic $32 \AA$ moiré pattern at the $2 \mathrm{D} / 3 \mathrm{D}$ interface. This moiré period is often hidden in conventional electron microscopy, where the Au structure is seen in projection. We show, via ab initio electronic structure calculations, that charge density is modulated according to the moiré period, illustrating the potential for (opto-) electronic moiré engineering at the 2D/3D interface. Our work presents a general pathway to directly image periodic modulation at interfaces using this combination of emerging microscopy techniques.

\footnotetext{
${ }^{1}$ Department of Materials Science and Engineering, Massachusetts Institute of Technology (MIT), Cambridge, MA, USA. ${ }^{2}$ Research Laboratory of Electronics, Massachusetts Institute of Technology (MIT), Cambridge, MA, USA. ${ }^{3}$ John A. Paulson School of Engineering and Applied Sciences, Harvard University, Cambridge, MA, USA. ${ }^{4}$ McGovern Institute for Brain Research, Massachusetts Institute of Technology, Cambridge, MA 02139, USA.

${ }^{5}$ Department of Physics and Astronomy, University of Victoria, Victoria, BC, Canada. ${ }^{6}$ Department of Brain and Cognitive Sciences, Massachusetts Institute of Technology, Cambridge, MA 02139, USA. ${ }^{7}$ These authors contributed equally: Kate Reidy, Georgios Varnavides. ${ }^{凶}$ email: fmross@mit.edu
} 
F ollowing the success of moiré engineering in modulating (opto-)electronic properties of graphene/hexagonal boron nitride (hBN) heterostructures ${ }^{1,2}$ and twisted bilayer graphene ${ }^{3-6}$, studies have extended the moiré toolbox to include systems such as double bilayer graphene ${ }^{7}$, trilayer graphene ${ }^{8}$, and van der Waals (vdW) heterostructures composed of transition metal dichalcogenides 9,10 and hBN-graphene-hBN stacks ${ }^{11,12}$. Recently, moiré engineering has been extended beyond vdW heterostructures, to $3 \mathrm{D} / 3 \mathrm{D}$ oxides ${ }^{13}$. Moiré engineering is yet to be utilized for tailoring the quasi-vdW interface between a $2 \mathrm{D}$ material and 3D metal. Engineering such 2D/3D interfaces is key to device applications where $2 \mathrm{D}$ materials make contact, through a well-controlled junction, to a $3 \mathrm{D}$ material such as a metal or semiconductor ${ }^{14-16}$. In contrast to $2 \mathrm{D} / 2 \mathrm{D}$ heterostructures, moiré engineering at the $2 \mathrm{D} / 3 \mathrm{D}$ interface requires consideration of the stacking of atomic planes in the out-of-plane direction. 3D stacking introduces an additional tuning parameter in $2 \mathrm{D} / 3 \mathrm{D}$ systems for modulating moiré properties that is not available in $2 \mathrm{D} / 2 \mathrm{D}$ heterostructures ${ }^{17}$.

The ability to image moiré superlattices directly is required to map electronic property modulation onto atomically-resolved structure $^{18}$. Various techniques have been used to observe moiré superlattices. These include reciprocal space imaging via low energy electron diffraction ${ }^{19,20}$ and convergent beam electron diffraction $(\mathrm{CBED})^{21}$; spatially resolved property measurement via scanning tunneling microscopy $(\mathrm{STM})^{22,23}$, atomic force microscopy (AFM) modalities 2,24 , near-field optical microscopy $^{13}$, and infrared nano-imaging 5 ; and imaging of transmitted intensity via high-resolution and dark field (scanning) transmission electron microscopy, (S)TEM ${ }^{25,26}$. Of these techniques, STM and (S)TEM are the only two that exhibit real-space atomic resolution. STM is widely used to characterize moiré patterns in 2D materials on bulk substrates, such as graphene on $\mathrm{Ru}^{27}, \mathrm{Ir}^{28}$, and $\mathrm{Cu}^{29}$. However, STM measurements are challenging for deeply buried interfaces and for the suspended layers that are gaining traction in $2 \mathrm{D}$ device physics ${ }^{30,31}$. (S)TEM, on the other hand, provides detailed information for suspended moiré systems fabricated from solely $2 \mathrm{D}$ materials ${ }^{25,26}$. Interpretation is more challenging for $2 \mathrm{D} / 3 \mathrm{D}$ interfaces due to the necessity of considering the $3 \mathrm{D}$ structure of layers away from the interface ${ }^{32,33}$. This has resulted in discrepancies in periodicity measurement between imaging techniques ${ }^{22,33}$. The $\mathrm{MoS}_{2} / \mathrm{Au}\{111\}$ system highlights these challenges, with different values reported for the periodicities of superlattices measured via STM and (S)TEM, $32 \AA$ and $18 \AA$ respectively ${ }^{22,33}$.

To reconcile such discrepancies and map moiré structureproperty relations at the $2 \mathrm{D} / 3 \mathrm{D}$ interface, we combine an analytic convolution technique and a range of STEM imaging techniques, integrated differential phase contrast (iDPC) and fourdimensional (4D) STEM, to decouple the spectrum of higher order moiré patterns. We investigate $\mathrm{MoS}_{2} / \mathrm{Au}\{111\}$ as a model 2D/3D system, relevant to TMDC (opto-) electronics ${ }^{14}$, and also examine hBN/Au $\{111\}$, relevant in plasmonics ${ }^{34,35}$. iDPC STEM measures the phase of the sample transmission function, enabling direct interpretation as the projected electrostatic potential in thin samples ${ }^{36-38}$. 4D STEM is a rapidly developing technique in which a pixelated array detector is used to collect a CBED pattern at each probe position in the STEM image. The resulting 4D dataset can be filtered post-acquisition to produce reconstructions such as bright field, annular bright field, annular dark field (ADF), ptychography, and iDPC ${ }^{39}$. 4D STEM has been applied to analysis of materials including $\mathrm{Cu}^{40}, \mathrm{ZrO}_{2}{ }^{41}, \mathrm{LiNiO}_{2}{ }^{42}$, $\mathrm{DyScO}_{3}{ }^{43}$, graphene ${ }^{44}, \mathrm{MoS}_{2}{ }^{45}$ and $\mathrm{WS}_{2}{ }^{46}$, with $2 \mathrm{D}$ materials particularly well-suited due to their small thickness ${ }^{47}$. We show that iDPC and 4D STEM are able to decouple higher order moiré periods to form real space images of the moiré pattern at the
2D/3D interface of $\mathrm{MoS}_{2} / \mathrm{Au}\{111\}$, revealing the crystallographic $32 \AA$ period. We explain the difference compared to conventional (S)TEM in terms of projection effects of the ABC stacking of the $3 \mathrm{D}$ metal. We then use ab initio electronic structure calculations to corroborate that $\mathrm{MoS}_{2} / \mathrm{Au}\{111\}$ charge density modulation is concentrated at the interface and follows the $32 \AA$ moiré periodicity. Together these findings demonstrate the utility of direct imaging via iDPC and 4D STEM for understanding the structure and electronic properties of 2D/3D heterostructures.

\section{Results}

Microscopy of the $\mathrm{MoS}_{2} / \mathbf{A u}\{111\}$ system. An example of the $\mathrm{MoS}_{2} / \mathrm{Au}\{111\}$ interface is shown in Fig. 1. In contrast to the mechanical transfer processes employed for fabricating vdW heterostructures, the 2D/3D systems studied here were formed by direct epitaxial growth ${ }^{48}$ in ultra-high vacuum conditions (Methods). The resulting samples consist of flat, faceted $\mathrm{Au}\{111\}$ nanoislands with an average edge length of $25 \mathrm{~nm}$ and height of $8 \mathrm{~nm}$ (Fig. 1a, Supplementary Fig. 1) that are epitaxially aligned on suspended $\mathrm{MoS}_{2}\{0001\}$ (Fig. 1b), with uniform moiré periodicities across micrometre-scale areas. Selected-area electron diffraction (SAED) confirms $0^{\circ}$ rotation between $\mathrm{Au}$ and $\mathrm{MoS}_{2}$ with a standard deviation of $0.2^{\circ}$ (Supplementary Fig. 2). In Fig. $1 \mathrm{~b}$ and other SAEDs, we observe spots indexed as $1 / 3\{422\} \mathrm{Au}$ reflections. These are classically forbidden for the FCC structure but their presence is consistent with $\mathrm{Au}$ nanoisland literature $\mathrm{r}^{49}$ (Supplementary Note 1). High resolution (HR) TEM shows that the islands are single crystalline, with no evidence of misfit dislocations and grain boundaries (Fig. 1c). The discontinuity in the moiré pattern visible at some boundaries arises from island coalescence. Here, both rigid body displacements and twin boundaries arise from stacking faults between coalesced islands (Fig. 1d, blue arrows).

The uniform moiré periodicity and sinusoidal intensity modulation show that the $\mathrm{Au}$ and $\mathrm{MoS}_{2}$ lattices are undistorted in the plane of the interface, even near island edges. This is different from the case of twisted vdW structures, which frequently display reconstructions ${ }^{25,26}$. The absence of distortion can likely be attributed to weak quasi-vdW bonding at the $\mathrm{MoS}_{2} / \mathrm{Au}\{111\}$ interface ${ }^{14}$. Motion of islands at room temperature is consistent in suggesting loose binding of the Au nanoislands to the underlying substrate (Supplementary Movie 1). During their motion, the islands exhibit rotation up to $0.3^{\circ}$, visually amplified in the angle of the moiré pattern (Supplementary Fig. 2). These $\mathrm{MoS}_{2} / \mathrm{Au}\{111\}$ interface characteristics are consistent for a range of Au thicknesses and uniform across samples (Supplementary Fig. 3).

Moiré site inequivalence. At first glance, the period of the $\mathrm{MoS}_{2} /$ $\mathrm{Au}\{111\}$ moiré superlattice in Fig. 1c, $d$ is $18 \AA$. While this is in agreement with previous HRTEM studies ${ }^{33}$, it is a consequence of the projective nature of conventional (S)TEM imaging. To illustrate this, one can consider a thought-experiment in which the out-of-plane coordinate of the 3D Au$\{111\}$ structure is ignored; this results in a "projected" hexagonal $\mathrm{Au}$ lattice with atomic spacing of $1.66 \AA$, which indeed yields a moiré pattern of $18 \AA$ with the $\mathrm{MoS}_{2}$ substrate. A more accurate view of electron scattering through the Au crystal requires us to include the full facecentred cubic (FCC) Au structure, as shown in Fig. 2a, b. Consider a location where an $\mathrm{Au}$ atom from the A layer (orange) is directly above a pair of $S$ atoms, as in the centre of Fig. 2c-top. This site repeats every $32 \AA$, shown by the orange squares in Fig. 2b. Sites that appear similar (red and blue squares in Fig. 2b) instead have $\mathrm{Au}$ atoms from the $\mathrm{B}$ or $\mathrm{C}$ layers above the $\mathrm{S}$ atoms (Fig. 2c-middle, 2c-bottom). The inequivalence of the three sites can be further illustrated via radial distribution functions (RDFs), 


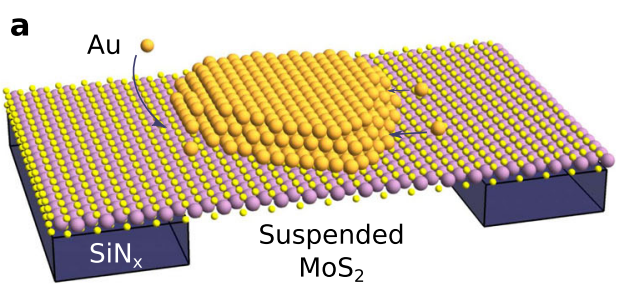

b
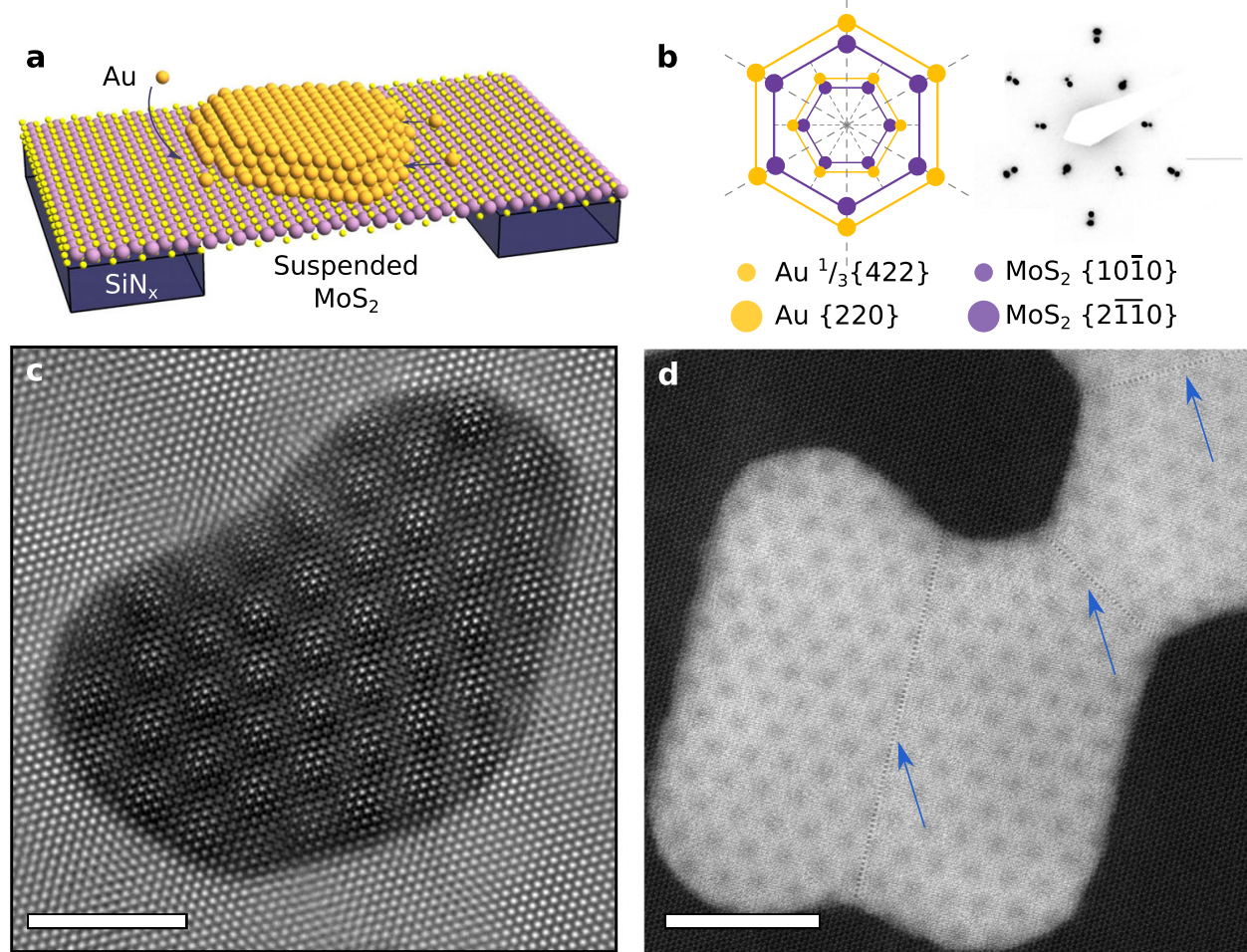

$\mathrm{Au}\{220\}$

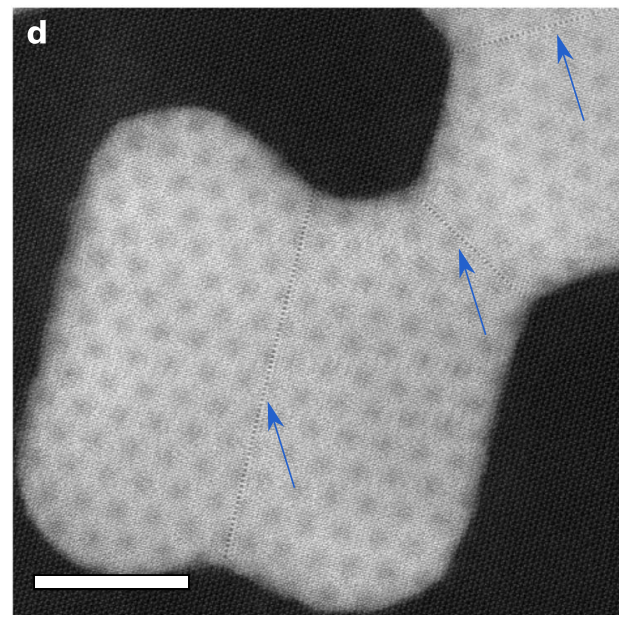

Fig. 1 HRTEM and STEM demonstrating epitaxial $\mathbf{M o S}_{2} / \mathbf{A u}\{\mathbf{1 1 1}\}$ moiré. a Schematic of epitaxially aligned Au deposited on suspended $M_{0} S_{2}$ supported on a $\mathrm{SiN}_{\mathrm{x}}$ TEM grid. Orange atoms represent $\mathrm{Au}$, yellow $\mathrm{S}$, and purple Mo. The $\mathrm{SiN}_{\mathrm{x}}$ membrane is shown in dark blue. b Reciprocal space model and experimental selected area electron diffraction pattern of the $A u\{111\}$ zone aligned on $\operatorname{MoS}_{2}\{0001\}$, with weak intensity $1 / 3\{422\}_{\text {Au }}$ spots (see text) aligned with $\{10 \overline{1} 0\}_{\mathrm{MoS}_{2}}$ and higher intensity $\{220\}_{\mathrm{Au}}$ spots aligned with $\{2 \overline{1} 10\}_{\mathrm{MoS}_{2}}$. Orange dots represent frequencies from Au crystal planes, while purple represent frequencies from $\mathrm{MoS}_{2}$ crystal planes. c HRTEM image of Au nanoisland on $\mathrm{MoS}_{2}$, showing apparent $18 \AA$-period moiré pattern. Scale bar, $40 \AA$. d High angle annular dark field (HAADF) STEM image. Scale bar, $80 \AA$. Coalescence boundaries are marked by blue arrows.

which show the quantitative difference in atomic locations (Fig. 2d). Although HRTEM (Fig. 1c) and STEM (Figs. 1d, 2e, g) do not distinguish the three sites, we find that iDPC STEM imaging (Fig. 2f), sensitive to the projected electrostatic potential $^{50,51}$, shows small changes in contrast that are statistically significant (Fig. 2h, Methods) and are confirmed by multislice simulations (Supplementary Fig. 4). iDPC can therefore detect the true $32 \AA$ moiré cell at the $\mathrm{MoS}_{2} / \mathrm{Au}\{111\}$ interface. However, although this modulation is qualitatively and statistically observable, the translation and rotation of the quasi-vdW islands, as in Supplementary Movie 1, preclude a quantitative analysis.

Although visible, the iDPC signal from the $32 \AA$ moiré is weak. To consider the full set of spatial frequencies of the Au\{111\} FCC crystal and obtain a clear real-space image of the $32 \AA$ moiré, we turn to a reciprocal space convolution theorem to predict the entire spectrum of possible moirés in the $\mathrm{MoS}_{2} / \mathrm{Au}\{111\}$ system (Methods). The geometric interpretation of the convolution theorem indicates that periodicities arise from the pairwise vectors connecting all spatial frequencies of the $\mathrm{MoS}_{2}$ and $\mathrm{Au}$ lattices $^{52}$ (Fig. 3a). In Supplementary Fig. 5 and Supplementary Table 1, we calculate these periodicities and intensities as a function of rotation angle between the two crystals. The four largest periodicities are shown in Fig. 3b. Additional higher order moirés are also predicted which often exhibit smaller periodicities and weaker intensities (Supplementary Fig. 5). At zero rotation, we indeed recover the $32 \AA$ moiré period, alongside the apparent $18 \AA$ moiré (Fig. 3b). Note that 32 A moiré periodicity is obscured by the $230 \%$ higher intensity reflections of the $18 \AA$ period convolution. We confirm this assignment of moiré periods by showing the experimental fast fourier transform (FFT) of Fig. 1c
(Methods, Fig. 3c). The moiré superlattice periods emerge as two sets of satellite peaks around the central beam spot. The simulated diffraction pattern in Fig. 3d is in quantitative agreement with the FFT of the acquired image (Fig. 3c), predicting all the higher order moiré periodicities at the $2 \mathrm{D} / 3 \mathrm{D}$ interface.

To extract a real space image of the weak $32 \AA$ moiré, we employ the technique of 4D STEM (Fig. 4a) ${ }^{39}$. Subsequently we select, with a virtual ADF detector, an annular area of each diffraction pattern to reconstruct an image from the average pattern (Fig. 4b) using certain diffraction spots only. Using an annulus that includes the $\mathrm{Au}\{220\}$ spots and the $\mathrm{MoS}_{2}\{2 \overline{11} 0\}$ spots (Fig. 4c, Methods), we observe the high intensity 18 A moiré pattern (Fig. 4e). The moiré shows uniform periodicity and sinusoidal intensity modulation across the islands. The symmetry is reduced to periodic line patterns in some areas due to sample tilt, but $18 \AA$ periodicity appears across all islands. If instead we generate a second virtual ADF image using the weaker ${ }^{1} / 3\{422\}$ $\mathrm{Au}$ and $\{10 \overline{1} 0\} \mathrm{MoS}_{2}$ reflections (Fig. 4d), we observe a hexagonal pattern of spots with $32 \AA$ moiré periodicity, consistent with our predictions from geometric convolution and the true crystallographic moiré accounting for 3D structure (Fig. 4f).

Charge density modulation. To explore the impact of the moiré periodicity on ground state charge density of our 2D/3D structure, we next turn to ab initio electronic structure calculations (Methods). Figure 5a shows a calculated isosurface of groundstate charge density difference for $\mathrm{MoS}_{2} / \mathrm{Au}\{111\}$ in side-view. The charge density difference is concentrated at the interface, specifically on the upper S layer of atoms, with some penetration to the underlying Mo layer. On the Au side, the charge density 
a

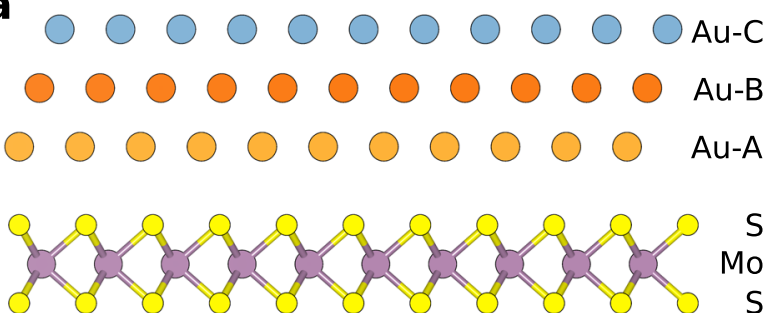

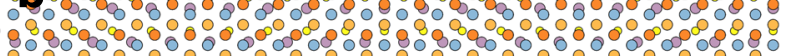

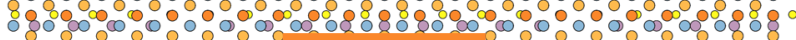

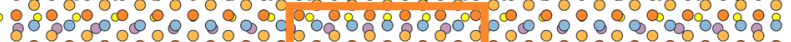

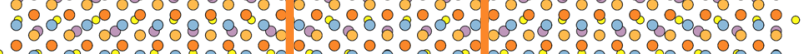

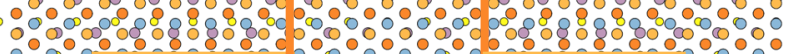

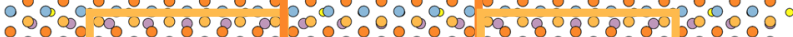

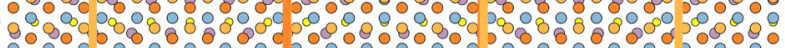

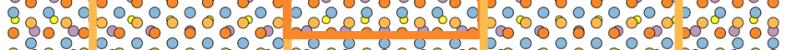

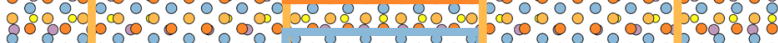

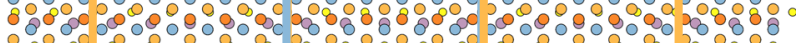

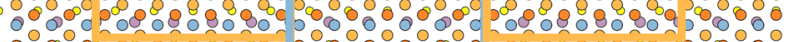

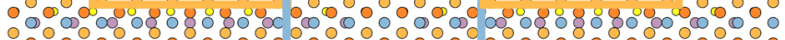

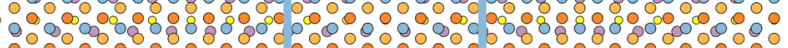

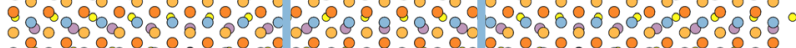

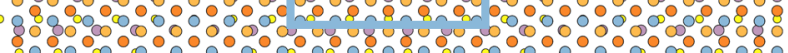

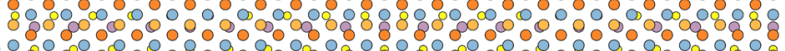

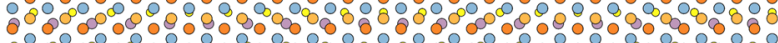

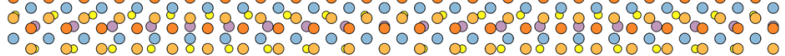

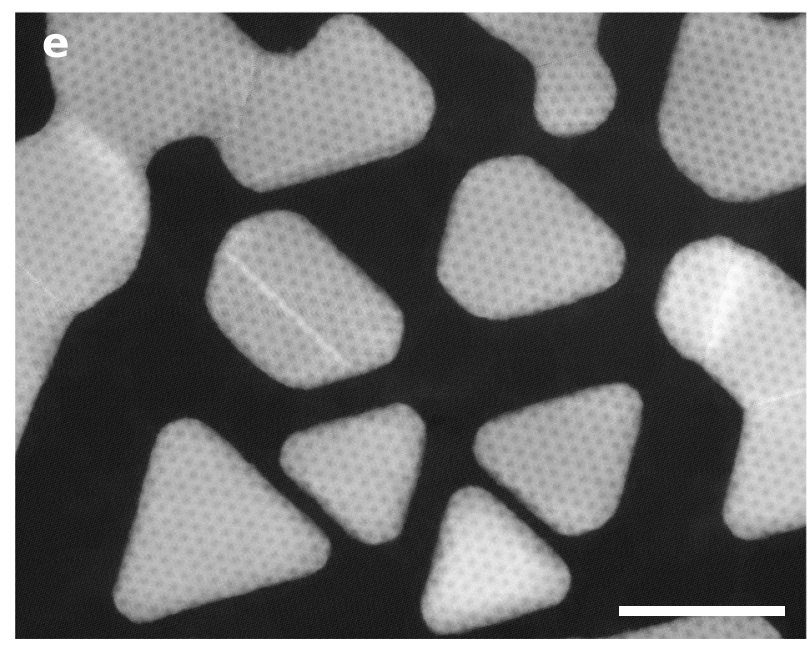

g
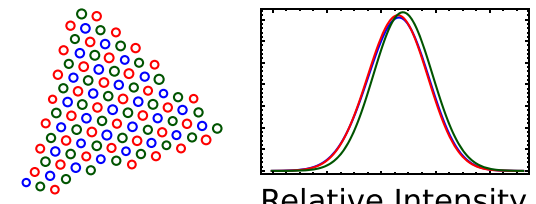

Relative Intensity

$=0.886$

$=0.099$

$=0.046$

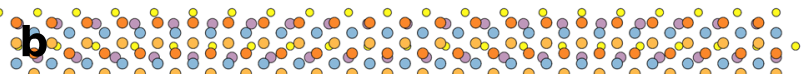
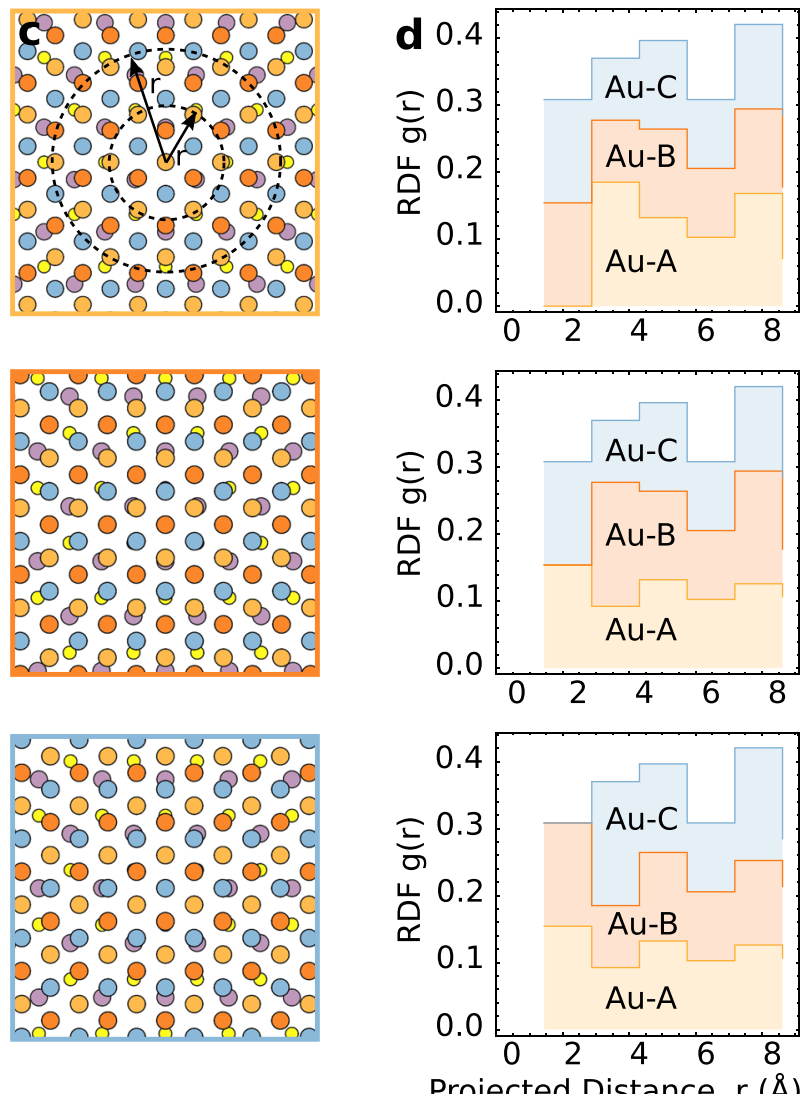

Projected Distance, $r(\AA)$

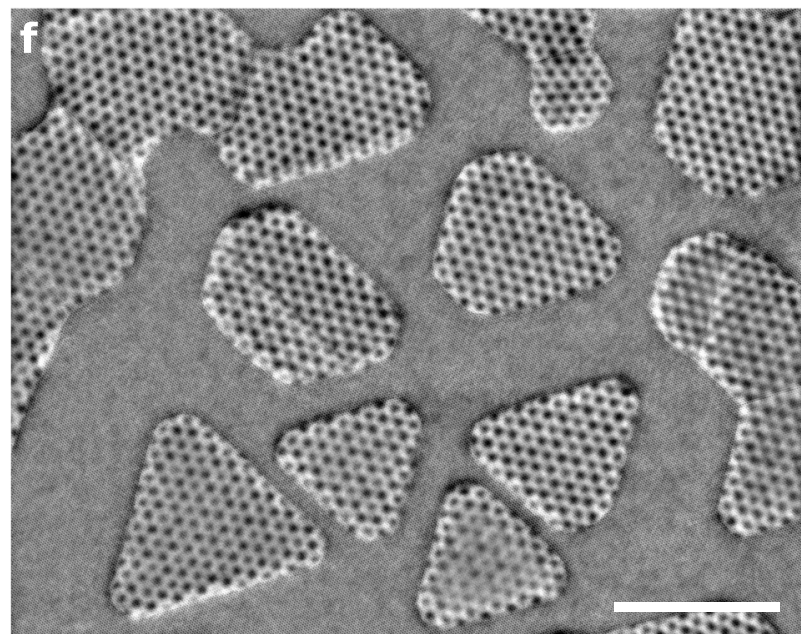

h
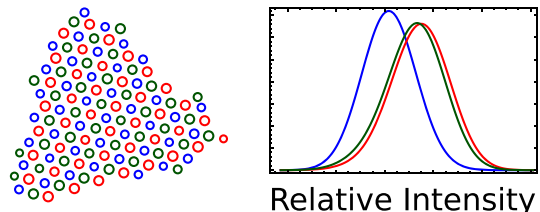

p-values

Fig. 2 Atomic models, RDFs, HAADF, and iDPC characterization of $32 \AA$ moiré structure. a Atomic model [100] zone axis for the $32 \AA$ moiré with the Au atoms indicated (orange, red, blue) to highlight relative stacking of $A, B$, and $C$ sites. $\mathbf{b}$ Plan view atomic model for the $32 \AA$ moiré. Boxed areas represent three inequivalent sites in the $32 \AA$ moiré. c Close up plan-view image of each of the sites highlighted in (b). $r$ is the projected distance from the central aligned sites, and the dotted circles show two representative $r$ values. d Corresponding RDFs of the three inequivalent sites in the $32 \AA$ moiré. e HAADF and $\mathbf{f}$ iDPC STEM images showing the (apparent) $18 \AA$ and $32 \AA$ moiré cells, respectively. Scale bars $200 \AA$. $\mathbf{g}$, h Relative intensity distributions and statistical variation of the three inequivalent sites in the island immediately above in the corresponding images. The equivalent disk radius for each spot was calculated and partitioned to inequivalent sites (red, green, blue). The histograms were smoothed using a gaussian kernel of radius $0.5 \AA$ for visual clarity. 

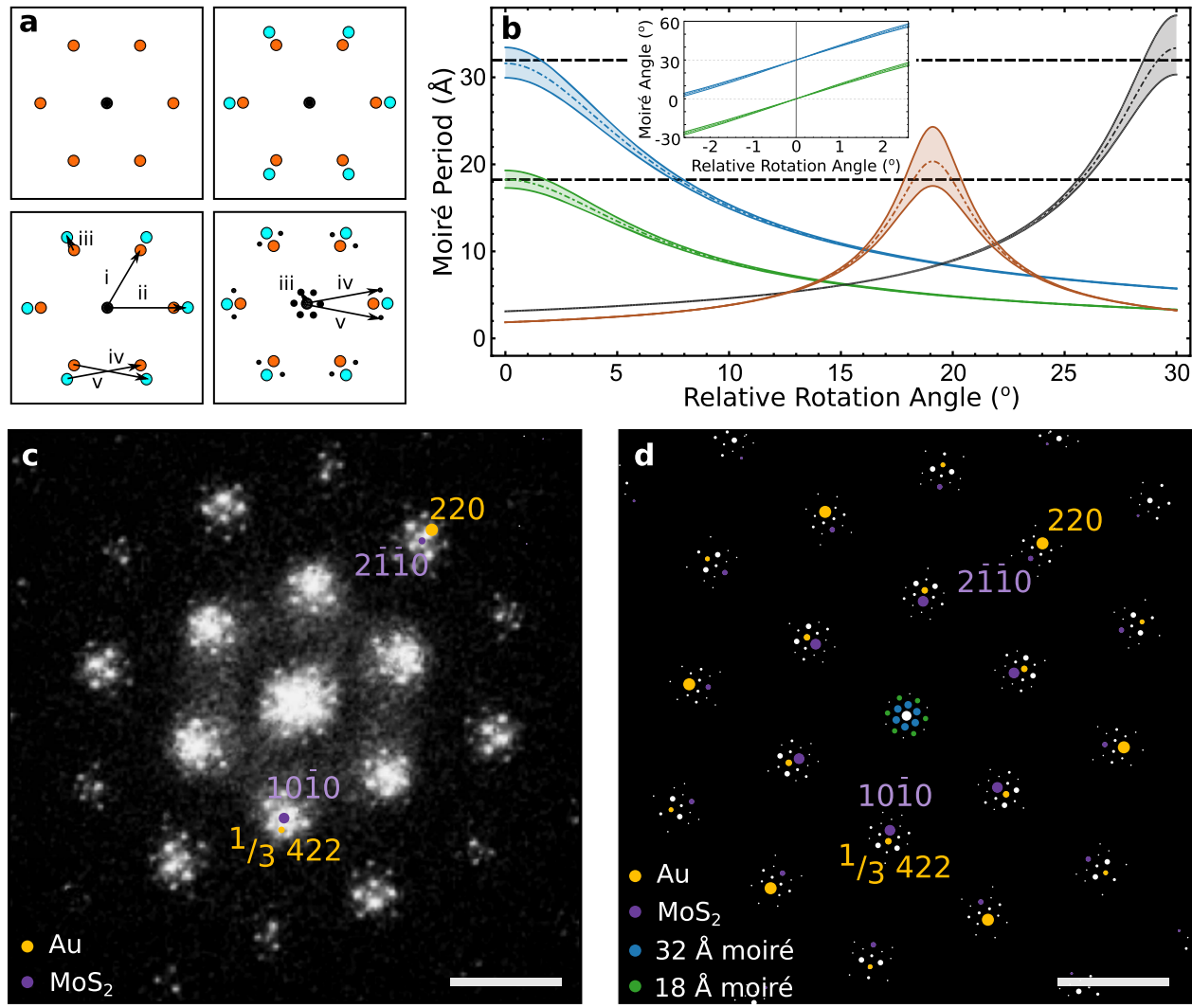

Fig. 3 Geometric convolution technique to predict moiré spectrum. a Schematic representation of satellite spot generation. Spatial frequencies due to a single lattice shown in the top left panel (orange) are overlaid on those arising from a second lattice on the top right (cyan). The convolution of these two sets of spatial frequencies ( $i$, ii) can be understood as the pairwise vectors connecting spatial frequencies of the two lattices (iii, iv, $v$ - bottom, left). These convolutions generate moiré frequencies (iii, iv, v) shown as black dots in the bottom right panel. $\mathbf{b}$ Calculated moiré period vs rotation angle for the four largest moiré supercells in the $\mathrm{MoS}_{2} / \mathrm{Au}\{111\}$ system, illustrated for small $( \pm 1 \%)$ Au lattice strain. Dot dashed lines represent $0 \%$ strain, while the two solid lines on either side represent \pm 1 strain as a bound. Black dashed lines represent the experimentally observed moiré periods from the FFT, two of which $\left(18 \AA\right.$ and $32 \AA$ ) are predicted at $0^{\circ}$ relative rotation angle. The moirés are color coded according to the reflections they arise from, with blue arising from the $\{2 \overline{11} 0\}_{\mathrm{MoS}_{2}}:\{220\}_{\mathrm{Au}^{\prime}}$ green $\{10 \overline{10}\}_{\mathrm{MoS}_{2}}: 1 / 3\{422\} \mathrm{Au}$, orange $\{6 \overline{330}\}_{\mathrm{MoS}_{2}}:\{642\}_{\mathrm{Au}}$ and grey $\{20 \overline{2} 0\}_{\mathrm{MoS}_{2}}:\{220\}_{\mathrm{Au}}$ reflections, respectively. The inset shows the variation of moire angle with relative rotation angle near $0^{\circ}$. c FFT of atomic resolution HRTEM image of the $\mathrm{MoS}_{2} / \mathrm{Au}\{111\}$ image in Fig. $1 \mathrm{c}$ showing $1 / 3\{422\}$ reflection and two visible moiré periodicities around the central spot. Illustrative orange dots represent frequencies from Au crystal planes, while purple represent frequencies from $\mathrm{MoS}_{2}$ crystal planes. Scale bar, $0.5 \AA^{-1}$. d Simulated FFT for Au/MoS 2 generated via the geometric convolution technique with each spot colored to show its origin (orange: $A$ u, purple: $\mathrm{MoS}_{2}$, blue: $32 \AA$ crystallographic moiré, green: apparent $18 \AA$ moiré). Area of spots is proportional to absolute intensity, but with inner moiré spots magnified $2 x$ for clarity. Scale bar, $0.5 \AA^{-1}$.

difference is concentrated on the first atomic plane, with negligible charge density found in the second $\mathrm{Au}\{111\}$ layer. The charge density modulation due to the $2 \mathrm{D} / 3 \mathrm{D}$ interface indeed has a periodicity of $32 \AA$ (Fig. 5b). To quantify the effect of moiré modulation on band structure and density of states, the supercell electronic states can be unfolded onto a single $\mathrm{MoS}_{2}$ unit cell (Fig. 5c). Accounting for the $32 \AA$ moiré, band structure calculations are in agreement with prior angle resolved photoemission spectroscopy and scanning tunnelling spectroscopy measurements of the $\mathrm{MoS}_{2} / \mathrm{Au}\{111\}$ system ${ }^{53}$.

Application of the method to the $\mathrm{hBN} / \mathrm{Au}\{111\}$ interface. To explore the generality of the geometric convolution technique, we also apply it to the hBN/Au $\{111\}$ structure (Supplementary Fig. 6). Here, the $\mathrm{Au}$ lattice is rotated by $10^{\circ}$ with respect to the $\mathrm{hBN}$, leading to a more complex situation than the symmetric $0^{\circ}$ epitaxy of $\mathrm{MoS}_{2} / \mathrm{Au}\{111\}$. This $10^{\circ}$ rotation leads to a strong moiré periodicity of $11 \AA$. Prediction via geometric convolution technique is necessary in these rotated systems to uncover higher order moirés. For this interface, the convolution technique predicts and explains an additional $19 \AA$ periodicity observed experimentally and re-creates the experimental diffraction pattern (Supplementary Fig. 6). The predictions of the convolution technique rely solely on inputs of crystal structure, lattice parameters, and rotation. This analysis, as well as previous literature, illustrate the wide applicability of the technique in moiré analysis ${ }^{52}$.

\section{Discussion}

For the $\mathrm{MoS}_{2} / \mathrm{Au}\{111\}$ interface, the combination of several different imaging techniques with electronic calculations provides a clearer picture of the moiré structure than is possible with any single measurement. In HRTEM the $18 \AA$ moiré is the strongest visible, leading to the possibility of erroneously predicting that electronic properties should be modulated with this period. Our calculations reveal that electronic modulation instead follows the true crystallographic $32 \AA$ periodicity. This periodicity is hidden in conventional TEM due to projection effects. Instead, for this interface, 4D STEM imaging, combined with a geometric convolution analysis of the full moiré spectrum, allows a direct realspace observational link between atomic structure and moiréinduced electronic modulation at this $2 \mathrm{D} / 3 \mathrm{D}$ interface. The combination of analysis techniques also explains the discrepancy 

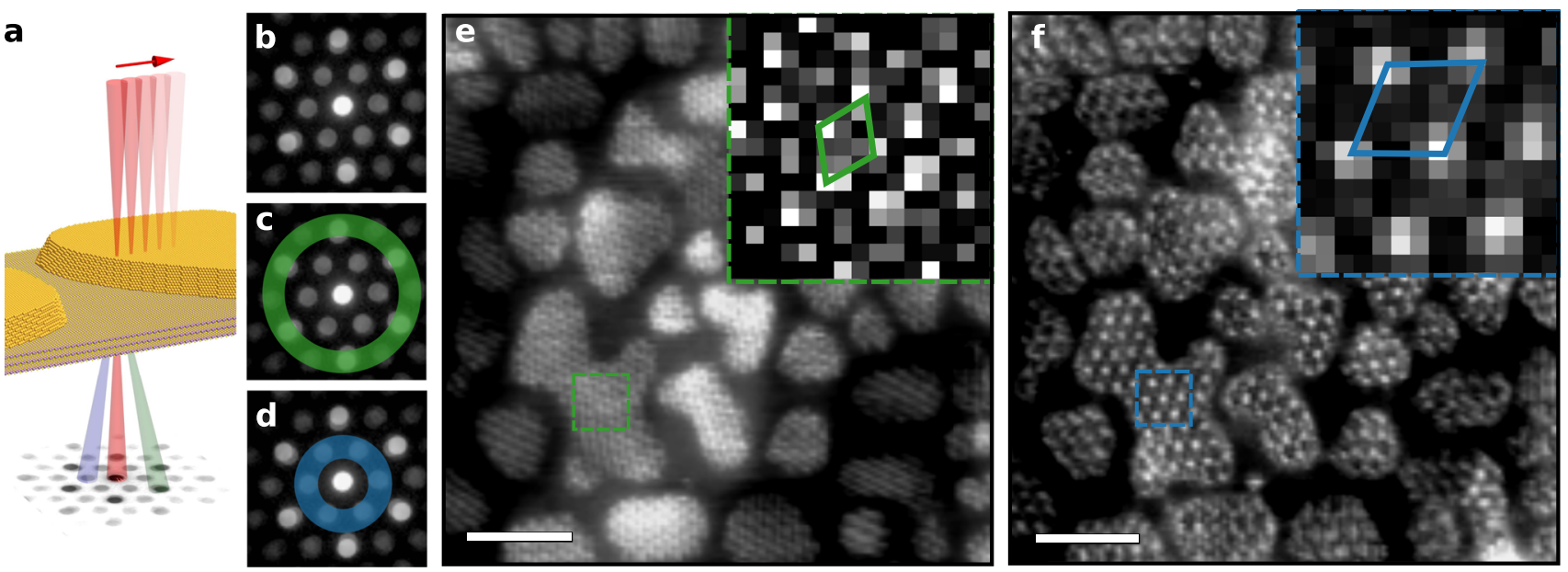

Fig. 4 4D STEM imaging of $18 \AA$ and $32 \AA$ moiré periodicities. a Schematic of $4 D$ STEM technique showing rastered beam (red) on MoS $2 / A u\{111\}$ with corresponding CBED pattern at each point. The green and blue scattered beams are centred on the spots of the annuli shown in (c and $\mathbf{d}$ ). $\mathbf{b}$ CBED pattern formed by averaging patterns collected over the entire scan area, c 4D STEM annulus used to isolate $18 \AA$ moiré periodicity (angular range 31-43 mrad, green) and $\mathbf{d}$ 4D STEM annulus used to isolate $32 \AA$ periodicity (angular range 11-24 mrad, blue). e, f Virtual ADF STEM images revealing 18 (green) and $32 \AA$ (blue) period moirés, respectively. Scale bar, $200 \AA$. Insets show unit cells.
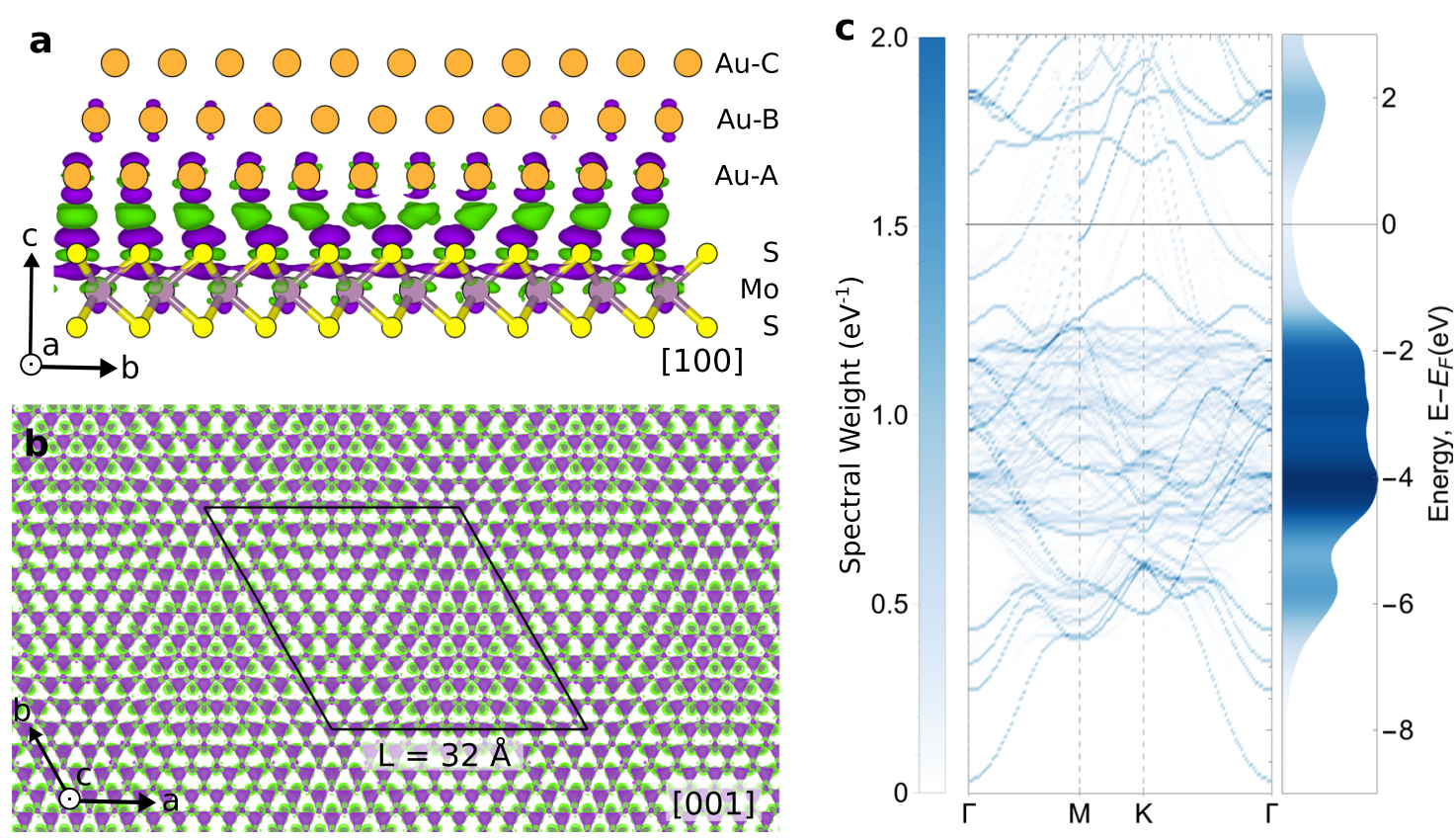

Fig. 5 Electronic structure calculations at the 2D/3D interface. a Charge density difference viewed down a [100] cross section of the $32 \AA$ commensurate moiré $\left(11_{\mathrm{Au}} \times 10_{\mathrm{MoS}_{2}}\right.$ superstructure). Orange atoms represent Au, yellow $\mathrm{S}$, and purple Mo. Purple denotes negative and green positive charge density isosurface contours. b Calculated charge density difference at the $\mathrm{MoS}_{2} / \mathrm{Au}\{111\}$ interface, as viewed down the [001] axis, showing electronic modulation following the $32 \AA$ moiré periodicity. Black line indicates the $32 \AA$ crystallographic moiré unit cell. c Unfolded band structure for MoS $2 / A u\{111\}$ system (left) and corresponding density of states (right). Color corresponds to the band's spectral weight.

between moiré patterns observed by TEM and STM at this $2 \mathrm{D} / 3 \mathrm{D}$ interface. These results highlight electronic modulation at the $2 \mathrm{D} / 3 \mathrm{D}$ interface, and showcase the growing opportunities for advanced STEM techniques for direct imaging of moiré structures at the atomic scale.

We envision that the coupled application of 4D STEM and the geometric convolution theorem, presented here for analysis of the $2 \mathrm{D} / 3 \mathrm{D}$ interface, could also be extended to the direct imaging of higher order moirés in systems with multiple interfaces and could expand opportunities across the field of moiré engineering. Potential applications lie in multiple overlaid moiré superlattices, which have been found to coexist in vdW heterostructures such as hBN-graphene-hBN stacks"11,12, or in so-called "moiré of moirés" structures arising from relaxation of twisted trilayer graphene and $\mathrm{WSe}_{2}{ }^{54}$. Although the effects of these coexisting moirés have been reported, they have not yet been directly imaged. This is because the overall moiré observed in HRTEM and STEM is a convolved projection of all the moirés in the system. Using 4D STEM and geometric convolution, moiré characterization could in theory be performed at each interface in the structure by highlighting the relevant diffraction spots. Virtual ADF images could then be used to decouple and directly image each separate moiré. Moreover, 4D STEM could enable simultaneous mapping of crystal orientation, strain, sample thickness, 
polarization, electric fields, and 3D ptychographic reconstructions of relevant moiré structures ${ }^{39}$. To date, most $2 \mathrm{D} / 3 \mathrm{D}$ moiré investigations (including this study) have focussed on epitaxially grown interfaces exhibiting a single orientation. However, future practical development of $2 \mathrm{D} / 3 \mathrm{D}$ moiré engineering will require complete control of the structure and orientation of $2 \mathrm{D}$ and $3 \mathrm{D}$ materials. Emerging fabrication methods using direct transfer of a $3 \mathrm{D}$ metal, such as $\mathrm{Au}$, onto $2 \mathrm{D}$ materials ${ }^{55}$, or nanomechanical rotation of a $3 \mathrm{D}$ nanocrystal using AFM or STM cantilevers ${ }^{56}$, suggest that such control of interfacial orientation is increasingly feasible, extending opportunities of 2D/3D moiré engineering.

\begin{abstract}
Methods
Suspended $\mathbf{M o S}_{\mathbf{2}}$ sample fabrication. Custom TEM chips were fabricated that include a $\mathrm{SiN}_{\mathrm{x}}$ membrane supported on $\mathrm{Si}$, with 9 holes each $4 \mu \mathrm{m}$ in diameter. We employed a wedging transfer process to suspend $\mathrm{MoS}_{2}$ on these TEM grids ${ }^{57}$. Thermally grown $90 \mathrm{~nm} \mathrm{SiO} / / \mathrm{Si}$ wafers were pre-treated with oxygen plasma and $\mathrm{MoS}_{2}$ was mechanically exfoliated onto them using the conventional Scotch tape method. Flakes of suitable thickness were identified by their contrast in optical microscopy. A solution of $25 \mathrm{~g}$ cellulose acetate butyrate (CAB) in $100 \mathrm{ml}$ ethyl acetate was spin coated onto the sample and baked at $80^{\circ} \mathrm{C}$ for $6 \mathrm{~min}$. MoS $\mathrm{S}_{2}$ flakes were transferred to the TEM grids using a wedging transfer technique ${ }^{57}$. Here, a scalpel is used to cut the $\mathrm{CAB}$ around the desired flake. A drop of deionised water can then be intercalated between the $\mathrm{CAB}$ and $\mathrm{SiO}_{2} / \mathrm{Si}$ surface and the entire flake transferred to the TEM grid with the CAB polymer handle using a tweezers. The transferred flakes were baked at $140^{\circ} \mathrm{C}$ for $5-10 \mathrm{~min}$ to improve adhesion. After dissolving the $\mathrm{CAB}$ in acetone for $15 \mathrm{~min}$, the flakes were dipped in isopropanol and dried using a critical point dryer.
\end{abstract}

Ultra-high vacuum (UHV) epitaxial deposition. To create epitaxial nanoislands, UHV deposition is used. This reduces impurities trapped at the metal-2D interface ${ }^{58}$. The main source of interfacial impurities is polymer residues, which create heterogeneous nucleation sites. Therefore, polymer residue remaining on the 2D material nucleates non-epitaxially aligned nanoislands (Supplementary Fig. 1). The combination of $\mathrm{CAB}$ polymer and heat treatment is effective in removing carbon and polymeric contamination ${ }^{31}$; material transferred using other polymers such as PMMA cannot be cleaned as effectively. $\mathrm{MoS}_{2} / \mathrm{SiN}_{\mathrm{x}}$ substrates were loaded into a UHV sample preparation chamber and cleaned of residual polymer by heating resistively in UHV to $\sim 550^{\circ} \mathrm{C}$ for several hours. Au deposition was carried out in the same multichamber UHV system (base pressure $2 \times 10^{-9}$ Torr), and was deposited in a homebuilt K-cell, using sheet metal placed in a $\mathrm{BN}$ crucible, at a rate of $0.5 \AA / \mathrm{min}$. The deposited thickness was calibrated by measuring the evaporation rate with a quartz crystal microbalance immediately before and after deposition. AFM analyses of island thickness were performed in a Veeco Metrology Nanoscope $\mathrm{V}$ in tapping mode. There is no intentional heating during deposition, but thermocouple measurements show that the sample temperature rises to $50-60{ }^{\circ} \mathrm{C}$

TEM imaging and data analysis. A field-emission TEM (JEOL 2010F) was used for selected area electron diffraction and bright-field imaging, operated at $200 \mathrm{kV}$. HRTEM imaging was performed with a Hitachi HF-3300V with CEOS BCOR imaging aberration corrector, operated at $60 \mathrm{kV}$. Figure $1 \mathrm{c}$ was obtained from a drift corrected mean of 25 images, where each image was an 8 second exposure, so the total exposure on to the camera was 200 seconds. The electron flux was $500 \mathrm{e}^{-} / \AA^{2} / \mathrm{sec}$ so the final image exhibited a total $\sim 100,000 \mathrm{e}^{-} / \AA^{2}$. Drift tracking over the images gave an average drift of $<7 \mathrm{pm} / \mathrm{sec}$, although most images exhibited less drift. FFTs and line-scans were obtained using Fiji ImageJ software. FFTs of the real-space image are used for observing moiré peaks instead of SAED patterns since moiré peaks were not readily observed at the energies $(80-300 \mathrm{keV})$ used in TEM $^{59}$. The FFT in Fig. $3 \mathrm{c}$ was produced by multiplying the source image (Fig. 1c) by a Hanning window prior to taking its FFT to minimise streaking incurred due to the hard edge of the image.

Multislice image simulations. STEM image simulations in Supplementary Fig. 4 were performed using an orthorhombic supercell consisting of $3 \mathrm{Au}$ layers on an $\mathrm{MoS}_{2}$ monolayer (7956 atoms), sliced along the [001] direction. A repeating unit from the supercell was cropped and simulated using custom Python-based STEM image simulation software. Simulation parameters similar to experiments were used, with an accelerating voltage of $60 \mathrm{kV}$, convergence angle of $24.7 \mathrm{mrad}$, and collection angles of 25-153 mrad (ADF) and 6-24 (iDPC). Simulated ADF and iDPC images were convolved with a gaussian kernel having FWHM of $80 \mathrm{pm}$, approximately accounting for the finite effective source size.

4D STEM imaging and data analysis. 4D STEM imaging was performed with a probe-corrected Thermo Fisher Scientific Themis Z G3 60-300 kV S/TEM operated at $60 \mathrm{kV}$ with a beam current of $50-60 \mathrm{pA}$ in the microprobe mode and a semiconvergence angle of $5.42 \mathrm{mrad}$, using an Electron Microscopy Pixel Array
Detector. The equivalent probe size used in Fig. 4 was $\sim 1 \mathrm{~nm}$ and the pixel size was $0.813 \mathrm{~nm}$. Virtual ADF STEM images were generated from the 4D STEM dataset using virtual detectors in the ' $4 \mathrm{D}$ STEM Explorer' $\operatorname{program}^{60}$. The HAADF and iDPC images in Fig. 2 were acquired at $200 \mathrm{kV}, 25 \mathrm{mrad}$ convergence angle, and a current of $30 \mathrm{pA}$. The quantification in Fig. 2g,h was performed as follows: input images were convolved with the Laplacian of a gaussian kernel with radius $3.75 \AA$ prior to peak detection; peaks were segmented using a watershed transform and an equivalent disk radius for each spot was calculated and partitioned to inequivalent sites (red, green, blue); a Student $t$-test was used to test the null hypothesis that the different sample means were equal, at the 0.001 significance level.

Geometric convolution technique. The geometric convolution code was implemented in the computational package Wolfram Mathematica 12.0 and builds on a model previously described for hexagonal lattices ${ }^{52}$. Frequencies arising from the superposition of the two lattice functions were obtained by the convolution theorem, $\mathrm{F}\{\mathrm{t} \mathrm{x} \mathrm{b}\}=\mathrm{F}\{\mathrm{t}\} \otimes \mathrm{F}\{\mathrm{b}\}$, where $\mathrm{t}$ and $\mathrm{b}$ are the top and bottom lattice functions respectively, F \{\} denotes the Fourier transform, and $\otimes$ denotes the convolution operation. All possible spatial frequencies arising from observed spots in the SAED/ FFT were initially obtained and we make no assumptions in the simulation other than the bulk structure of $\mathrm{Au}$ and its $\{111\}$ orientation. The full set of spatial frequencies of the FCC crystal along the [111] zone axis were used to calculate the moiré periods for all possibilities within the experimentally observed FFT as a function of the relative rotation, while allowing for small $( \pm 1 \%)$ Au lattice strain The angles can also be calculated (Supplementary Fig. 2). We then evaluate the most likely candidates to explain the experimentally measured moiré periods and angles (Supplementary Fig. 5).

Electronic structure calculations. The ground-state charge density difference $(\Delta \rho)$ between the $\mathrm{Au} / \mathrm{MoS}_{2}$ heterostructure $\left(\rho_{\mathrm{Au} / \mathrm{MoS}_{2}}\right)$, and pristine $\mathrm{Au}\left(\rho_{\mathrm{Au}}\right)$ and $\mathrm{MoS}_{2}\left(\rho_{\mathrm{MoS}_{2}}\right)$ is given by

$$
\Delta \rho=\rho_{\mathrm{Au} / \mathrm{MoS}_{2}}-\rho_{\mathrm{Au}}-\rho_{\mathrm{MoS}_{2}}
$$

Density functional theory calculations were carried out using the projector augmented wave method implemented in the Vienna ab initio simulation package, VASP ${ }^{61,62}$. We account for the vdW dispersion interactions using the generalized gradient optB86b-vdW functional ${ }^{63}$. We use a cut-off energy of $400 \mathrm{eV}$ on an equivalent Monkhorst-Pack k-points grid of 40x40x1 $\mathrm{MoS}_{2}$ unit cell (and similar density supercell). Bandstructure unfolding was performed using the BandUP $\operatorname{code}^{64}$.

\section{Data availability}

The authors declare that the main data supporting the findings of this study are available within the article and its Supplementary Information files.

\section{Code availability}

Code available upon request from the authors.

Received: 22 December 2020; Accepted: 20 January 2021; Published online: 26 February 2021

\section{References}

1. Dean, C. R. et al. Hofstadter's butterfly and the fractal quantum hall effect in moiré superlattices. Nature 497, 598-602 (2013).

2. Woods, C. R. et al. Commensurate-incommensurate transition in graphene on hexagonal boron nitride. Nat. Phys. 10, 451-456 (2014).

3. Cao, Y. et al. Correlated insulator behaviour at half-filling in magic-angle graphene superlattices. Nature 556, 80-84 (2018).

4. Hesp, N. C. H. et al. Collective excitations in twisted bilayer graphene close to the magic angle. Preprint at https://arxiv.org/abs/1910.07893 (2019).

5. Sunku, S. S. et al. Photonic crystals for nano-light in moiré graphene superlattices. Science 362, 1153-1156 (2018).

6. Balents, L., Dean, C. R., Efetov, D. K. \& Young, A. F. Superconductivity and strong correlations in moiré flat bands. Nat. Phys. 16, 725-733 (2020).

7. Liu, X. et al. Tunable spin-polarized correlated states in twisted double bilayer graphene. Nature 583, 221-225 (2020).

8. Chen, G. et al. Signatures of tunable superconductivity in a trilayer graphene moiré superlattice. Nature 572, 215-219 (2019).

9. Regan, E. C. et al. Mott and generalized Wigner crystal states in $\mathrm{WSe}_{2} / \mathrm{WS}_{2}$ moiré superlattices. Nature 579, 359-363 (2020).

10. Karni, O. et al. Infrared interlayer exciton emission in $\mathrm{MoS}_{2} / \mathrm{WSe}_{2}$ heterostructures. Phys. Rev. Lett. 123, 247402 (2019).

11. Finney, N. R. et al. Tunable crystal symmetry in graphene-boron nitride heterostructures with coexisting moiré superlattices. Nat. Nanotechnol. 14, 1029-1034 (2019) 
12. Wang, L. et al. New generation of Moiré superlattices in doubly aligned hBN/ Graphene/hBN heterostructures. Nano Lett. 19, 2371-2376 (2019).

13. Chen, X. et al. Moiré engineering of electronic phenomena in correlated oxides. Nat. Phys. 16, 631-635 (2020).

14. Allain, A., Kang, J., Banerjee, K. \& Kis, A. Electrical contacts to twodimensional semiconductors. Nat. Mater. 14, 1195-1205 (2015).

15. Bae, S.-H. et al. Integration of bulk materials with two-dimensional materials for physical coupling and applications. Nat. Mater. 18, 550-560 (2019).

16. Miao, J. et al. Gate-tunable semiconductor heterojunctions from $2 \mathrm{D} / 3 \mathrm{D}$ van der Waals interfaces. Nano Lett. 20, 2907-2915 (2020).

17. Wallbank, J. R., Mucha-Kruczyński, M. \& Fal'Ko, V. I. Moiré minibands in graphene heterostructures with almost commensurate $\sqrt{3} \times \sqrt{3}$ hexagonal crystals. Phys. Rev. B - Condens. Matter Mater. Phys. 88, 155415 (2013).

18. Luo, Y. et al. In situ nanoscale imaging of moiré superlattices in twisted van der Waals heterostructures. Nat. Commun. 11, 1-7 (2020).

19. Tiefenbacher, S., Pettenkofer, C. \& Jaegermann, W. Moire pattern in LEED obtained by van der Waals epitaxy of lattice mismatched WS2/MoTe2(0001) heterointerfaces. Surf. Sci. 450, 181-190 (2000).

20. de Jong, T. A. et al. Measuring the local twist angle and layer arrangement in van der Waals heterostructures. Phys. status solidi 255, 1800191 (2018).

21. Latychevskaia, T. et al. Convergent beam electron holography for analysis of van der Waals heterostructures. PNAS 115, 7473-7478 (2018).

22. Sørensen, S. G., Fü, H. G., Tuxen, A. K., Walton, A. S. \& Lauritsen, J. V. Structure and electronic properties of in situ synthesized single-layer $\mathrm{MoS}_{2}$ on a gold surface. ACS Nano 8, 6788-6796 (2014).

23. Kerelsky, A. et al. Maximized electron interactions at the magic angle in twisted bilayer graphene. Nature 572, 95-100 (2019).

24. McGilly, L. J. et al. Visualization of moiré superlattices. Nat. Nanotechnol. 15, 580-584 (2020).

25. Yoo, H. et al. Atomic and electronic reconstruction at the van der Waals interface in twisted bilayer graphene. Nat. Mater. 18, 448-453 (2019).

26. Weston, A. et al. Atomic reconstruction in twisted bilayers of transition metal dichalcogenides. Nat. Nanotechnol. 15, 592-597 (2020).

27. Sutter, P. W., Flege, J. I. \& Sutter, E. A. Epitaxial graphene on ruthenium. Nat. Mater. 7, 406-411 (2008).

28. Busse, C. et al. Graphene on $\operatorname{Ir}(111)$ : physisorption with chemical modulation. Phys. Rev. Lett. 107, 036101 (2011)

29. Gao, L., Guest, J. R. \& Guisinger, N. P. Epitaxial graphene on $\mathrm{Cu}(111)$. Nano Lett. 10, 3512-3516 (2010).

30. Zhou, Y. et al. Controlling excitons in an atomically thin membrane with a mirror. Phys. Rev. Lett. 124, 027401 (2020).

31. Thomsen, J. D. et al. Suppression of intrinsic roughness in encapsulated graphene. Phys. Rev. B 96, 1-8 (2017).

32. Tian, X. et al. Correlating the three-dimensional atomic defects and electronic properties of two-dimensional transition metal dichalcogenides. Nat. Mater. 19, 867-873 (2020).

33. Sun, Y. et al. Direct observation of epitaxial alignment of $\mathrm{Au}$ on $\mathrm{MoS}_{2}$ at atomic resolution. Nano Res. 12, 947-954 (2019).

34. Henrique, L. et al. Monolayer and thin hBN as substrates for electron spectromicroscopy analysis of plasmonic nanoparticles. Appl. Phys. Lett. 113, 231108 (2018).

35. Tran, T. T. et al. Deterministic coupling of quantum emitters in $2 \mathrm{D}$ materials to plasmonic nanocavity arrays. Nano Lett. 17, 2634-2639 (2017).

36. Dekkers, N. H. \& De Lang, H. Differential phase contrast in a STEM. OPTIK 41, (1974).

37. Lazić, I., Bosch, E. G. T. \& Lazar, S. Phase contrast STEM for thin samples: Integrated differential phase contrast. Ultramicroscopy 160, 265-280 (2016).

38. Müller, K. et al. Atomic electric fields revealed by a quantum mechanical approach to electron picodiffraction. Nat. Commun. 5, 1-8 (2014).

39. Ophus, C. Four-dimensional scanning transmission electron microscopy (4DSTEM): from scanning nanodiffraction to ptychography and beyond. Microsc. Microanalysis 25, 563-582 (2019).

40. Caswell, T. A. et al. A high-speed area detector for novel imaging techniques in a scanning transmission electron microscope. Ultramicroscopy 109, 304-311 (2009).

41. Watanabe, M. \& Williams, D. Development of diffraction imaging for orientation analysis of grains in scanning transmission electron microscopy. Microsc. Microanal. 13, 962-963 (2007).

42. Ahmed, S. et al. Visualization of light elements using 4D STEM: the layeredto-rock salt phase transition in $\mathrm{LiNiO}_{2}$ cathode material. Adv. Energy Mater. 10, $2001026(2020)$

43. Hachtel, J. A., Idrobo, J. C. \& Chi, M. Sub-Ångstrom electric field measurements on a universal detector in a scanning transmission electron microscope. Adv. Struct. Chem. Imaging 4, 1-10 (2018)

44. Ishikawa, R. et al. Direct electric field imaging of graphene defects. Nat. Commun. 9, 8-13 (2018).

45. Jiang, Y. et al. Electron ptychography of 2D materials to deep sub-ångström resolution. Nature 559, 343-349 (2018).
46. Fang, S. et al. Atomic electrostatic maps of $1 \mathrm{D}$ channels in $2 \mathrm{D}$ semiconductors using 4D scanning transmission electron microscopy. Nat. Commun. 10, 1-9 (2019).

47. Wen, Y. et al. Simultaneous identification of low and high atomic number atoms in monolayer $2 \mathrm{D}$ materials using $4 \mathrm{D}$ scanning transmission electron microscopy. Nano Lett. 19, 6482-6491 (2019).

48. Periwal, P. et al. Catalytically mediated epitaxy of 3D semiconductors on van der Waals substrates. Appl. Phys. Rev. 7, 31402 (2020).

49. Reyes-Gasga, J., Gómez-Rodríguez, A., Gao, X. \& José-Yacamán, M. On the interpretation of the forbidden spots observed in the electron diffraction patterns of flat Au triangular nanoparticles. Ultramicroscopy 108, 929-936 (2008).

50. Lohr, M. et al. Quantitative measurements of internal electric fields with differential phase contrast microscopy on InGaN/GaN quantum well structures. Phys. status solidi 253, 140-144 (2016).

51. Shibata, N. et al. Imaging of built-in electric field at a $\mathrm{p}-\mathrm{n}$ junction by scanning transmission electron microscopy. Sci. Rep. 5, 1-8 (2015).

52. Zeller, P. et al. What are the possible moiré patterns of graphene on hexagonally packed surfaces? Universal solution for hexagonal coincidence lattices, derived by a geometric construction. N. J. Phys. 16, 083028 (2014).

53. Bruix, A. et al. Single-layer $\mathrm{MoS}_{2}$ on $\mathrm{Au}(111)$ : band gap renormalization and substrate interaction. Phys. Rev. B 93, 165422 (2016).

54. Zhu, Z., Cazeaux, P., Luskin, M. \& Kaxiras, E. Modeling mechanical relaxation in incommensurate trilayer van der Waals heterostructures. Phys. Rev. B 101, 224107 (2020).

55. Went, C. M. et al. A new metal transfer process for van der Waals contacts to vertical Schottky-junction transition metal dichalcogenide photovoltaics. Sci. $A d v$. 5, eaax6061 (2019)

56. Ribeiro-Palau, R. et al. Twistable electronics with dynamically rotatable heterostructures. Science 361, 690-693 (2018).

57. Schneider, G. F., Calado, V. E., Zandbergen, H., Vandersypen, L. M. K. \& Dekker, C. Wedging transfer of nanostructures. Nano Lett. 10, 1912-1916 (2010).

58. English, C. D., Shine, G., Dorgan, V. E., Saraswat, K. C. \& Pop, E. Improved contacts to $\mathrm{MoS}_{2}$ transistors by ultra high vacuum deposition. Nano Lett. 16 , 47 (2016).

59. Latychevskaia, T., Escher, C. \& Fink, H.-W. Moiré structures in twisted bilayer graphene studied by transmission electron microscopy. Ultramicroscopy 197, 46-52 (2019).

60. LeBeau, J. M. 4D STEM Explorer. https://doi.org/10.5281/zenodo.1325482 (2018).

61. Kresse, G. \& Furthmüller, J. Efficient iterative schemes for ab initio totalenergy calculations using a plane-wave basis set. Phys. Rev. B 54, 11169-11186 (1996).

62. Joubert, D. From ultrasoft pseudopotentials to the projector augmented-wave method. Phys. Rev. B 59, 1758-1775 (1999).

63. Klime, J., Bowler, D. R. \& Michaelides, A. Van der Waals density functionals applied to solids. Phys. Rev. B 83, 195131 (2011).

64. Medeiros, P. V. C., Stafström, S. \& Björk, J. Effects of extrinsic and intrinsic perturbations on the electronic structure of graphene: retaining an effective primitive cell band structure by band unfolding. Phys. Rev. B 89, 041407 (2014).

\section{Acknowledgements}

This work was carried out with the use of facilities and instrumentation supported by NSF through the Massachusetts Institute of Technology Materials Research Science and Engineering Center DMR - 1419807. This work was carried out in part through the use of MIT.nano's facilities. G.V. and P.N. acknowledge funding from NSF Award DMR1905295 and from the Office of Naval Research (ONR) Grant Number N00014-18-12691 for the theory and computation in this work. P.N. is a Moore Inventor Fellow supported through Grant GBMF8048 from the Gordon and Betty Moore Foundation. K.R. acknowledges funding from a MIT MathWorks Engineering Fellowship and an OGE MIT Fellowship. J.D.T. acknowledges support from Independent Research Fund Denmark though Grant Number 9035-00006B. The authors would like to acknowledge Michael Tarkanian for help in manufacturing TEM sample holders; Professor Pierre Stadelmann, Dr. Ruud Tromp, and Dr. Qiong Ma for helpful discussions; a manuscript reviewer for the suggestion of additional iDPC measurements; and Profs. Jeehwan Kim and Silvija Gradecak for equipment access. This research was primarily conducted on the traditional, unceded territory of the Wampanoag Nation. We acknowledge the painful history of forced removal from this territory, and we respect the many diverse indigenous people connected to this land.

\section{Author contributions}

K.R., G.V. and F.M.R. conceived the project. K.R. and J.D.T. developed the epitaxial deposition and fabricated samples. G.V. extended the geometric convolution model in 2D/3D systems. K.R. and G.V. performed geometric convolution analysis and analysed the data. K.R. and J.D.T. performed SAED and bright field TEM imaging. 
A.M.B. performed $60 \mathrm{keV} \mathrm{keV} \mathrm{HRTEM} \mathrm{imaging.} \mathrm{A.K.} \mathrm{and} \mathrm{J.M.L.} \mathrm{performed} \mathrm{4D} \mathrm{STEM}$ imaging and STEM multislice simulations. K.R. performed 4D STEM data analysis, iDPC imaging, and multislice calculations with input from A.K. and T.P. J.D.T. performed AFM measurements. G.V. performed the density functional theory calculations and made atomic models, with input from P.N. and P.A. The manuscript was written with contributions from all authors.

\section{Competing interests}

The authors declare no competing interests.

\section{Additional information}

Supplementary information The online version contains supplementary material available at https://doi.org/10.1038/s41467-021-21363-5.

Correspondence and requests for materials should be addressed to F.M.R.

Peer review information Nature Communications thanks Jordi Arbiol and the other anonymous reviewer(s) for their contribution to the peer review of this work. Peer reviewer reports are available.
Reprints and permission information is available at http://www.nature.com/reprint

Publisher's note Springer Nature remains neutral with regard to jurisdictional claims in published maps and institutional affiliations.

(c) (i) Open Access This article is licensed under a Creative Commons Attribution 4.0 International License, which permits use, sharing, adaptation, distribution and reproduction in any medium or format, as long as you give appropriate credit to the original author(s) and the source, provide a link to the Creative Commons license, and indicate if changes were made. The images or other third party material in this article are included in the article's Creative Commons license, unless indicated otherwise in a credit line to the material. If material is not included in the article's Creative Commons license and your intended use is not permitted by statutory regulation or exceeds the permitted use, you will need to obtain permission directly from the copyright holder. To view a copy of this license, visit http://creativecommons.org/ licenses/by/4.0/.

(c) The Author(s) 2021 